\title{
CIENCIAMATRIA
}

Revista Interdisciplinaria de Humanidades, Educación, Ciencia y Tecnología

Año VII. Vol. VII. N¹2. Enero - Junio. 2021

Hecho el depósito de ley: pp201602FA4721

ISSN-L: 2542-3029; ISSN: 2610-802X

Universidad Nacional Experimental Francisco de Miranda (UNEFM). Santa Ana de Coro. Venezuela

Ángel Sebastián Martínez-Narváez; Juan Carlos Erazo-Álvarez; Cecilia Ivonne Narváez-Zurita

Cristián Andrés Erazo-Álvarez

DOI $10.35381 / \mathrm{cm} . v 7 i 12.450$

\section{Modelo de Gestión para un Centro de revisión técnico vehicular}

\section{Management Model for a Vehicle Technical Review Center}

Ángel Sebastián Martínez-Narváez

asmartinezn@est.ucacue.edu.ec

Universidad Católica de Cuenca, Cuenca

Ecuador

https://orcid.org/0000-0001-8562-3677

Juan Carlos Erazo-Álvarez

jcerazo@ucacue.edu.ec

Universidad Católica de Cuenca, Cuenca

Ecuador

https://orcid.org/0000-0001-6480-2270

Cecilia Ivonne Narváez-Zurita inarvaez@ucacue.edu.ec

Universidad Católica de Cuenca, Cuenca

Ecuador

https://orcid.org/0000-0002-7437-9880

Cristián Andrés Erazo-Álvarez

cristianerazo@ucacue.edu.ec

Universidad Católica de Cuenca, Cuenca

Ecuador

https://orcid.org/0000-0001-8746-4788

Recibido: 01 de octubre de 2020

Aprobado: 15 de diciembre de 2020 


\title{
RESUMEN
}

El objetivo de la presente investigación se basó en diseñar un modelo de gestión para la creación de un centro de revisión técnica vehicular para el cantón cañar que mejore la gestión técnica del área de matriculación. Tuvo primordialmente un alcance metodológico de tipo descriptivo con diseño no experimental transversal, realizada en una muestra poblacional de 89 que poseen un vehículo automotor y están sujetas a matricular y realizar revisión técnica vehicular en el cantón Cañar - Ecuador. De acuerdo con las encuestas realizadas sobre el proyecto de CRTV ubicado en la ciudad de Cañar. Se determinó que es necesario tres líneas de revisión para RTV, dos líneas para vehículos livianos y una línea mixta.

Descriptores: Administración pública; responsabilidad social; participación del público. (Palabras tomadas del Tesauro UNESCO).

\begin{abstract}
The objective of this research was based on designing a management model for the creation of a vehicle technical review center for the canton of Cañar that improves the technical management of the registration area. It had primarily a methodological scope of a descriptive type with a non-experimental cross-sectional design, carried out in a population sample of 89 who own a motor vehicle and are subject to registering and conducting a vehicle technical review in the canton of Cañar - Ecuador. According to the surveys carried out on the CRTV project located in the city of Cañar. It was determined that three check lines are needed for RTV, two lines for light vehicles and one mixed line.
\end{abstract}

Descriptors: Public administration; social responsibility; audience participation. (Words taken from the UNESCO Thesaurus). 


\section{CIENCIAMATRIA}

Revista Interdisciplinaria de Humanidades, Educación, Ciencia y Tecnología

Año VII. Vol. VII. No12. Enero - Junio. 2021

Hecho el depósito de ley: pp201602FA4721

ISSN-L: 2542-3029; ISSN: 2610-802X

Universidad Nacional Experimental Francisco de Miranda (UNEFM). Santa Ana de Coro. Venezuela

Ángel Sebastián Martínez-Narváez; Juan Carlos Erazo-Álvarez; Cecilia Ivonne Narváez-Zurita

Cristián Andrés Erazo-Álvarez

\section{INTRODUCCIÓN}

Los servicios públicos se diferencian de los privados en muchos aspectos principalmente en el interés para el cual fueron creados ya que los servicios públicos deben realizar énfasis en los siguientes aspectos: El objetivo de los servicios públicos debe ser el de maximizar el bien público. Debe garantizar la igualdad y equidad en su prestación. Se ofertan en régimen de monopolio u oligopolio. Atienden a todo el mercado cautivo, sin los mecanismos habituales de competencia. Las personas que reciben el servicio se los conocen como usuarios, los cuales no coinciden siempre con los clientes.

Al ser estos servicios de interés público, es necesario tomar en cuenta que las organizaciones gubernamentales que entregan servicios a la ciudadanía deben tomar conciencia sobre la eficacia donde se debe precautelar que el servicio tenga universalidad e igualdad y la eficiencia que es solidaridad y financiación subsidiada. Pero estos aspectos no son suficientes para obtener servicios de calidad dentro de una organización para ello es necesario tomar en cuenta tres elementos claves: trabajadores, infraestructura y organización del trabajo (Naranjo García, 2014).

La columna vertebral de toda institución, organización, para aportar al desarrollo económico, para mejorar la calidad de vida de las personas es indispensable una estructura organizacional adecuada y con los objetivos bien definidos, que permita para un mejor direccionamiento de los proceso, para así tener bien definidas las funciones laborales de todos y cada uno de los miembros de la misma. En el mundo actual donde se desarrollan los negocios es necesario y primordial que toda empresa, institución tengan bien definidos sus puestos de trabajo para facilitar el trabajo por parte de Gerencia, mediante una estructura organizacional clara y concisa esto generara un crecimiento sostenido, expansión por ende se incrementar sus ingresos que es el fin de toda empresa obtener ganancias y ofrecer un servicio de calidad a sus clientes, existen diferentes tipos de estructuras organizacionales por ende cada empresa deberá 
acoplarse a su necesidad o requerimiento, debido a que es un punto importante para el éxito según la toma de decisiones.

Es básico y primordial que toda empresa defina una estructura organizacional para el correcto funcionamiento de la cualquier empresa sin importar el tamaño de la misma y así poder ser rentables a corto plazo y ayudar a gerencia a identificar de mejor manera el personal que necesita la organización. Con una adecuada planificación se provee de recursos suficientes en la parte humana en la empresa, según el plan anual establecido, así también la importancia en las responsabilidades que corresponden a cada persona, lo que contribuye a un mejor desempeño de la empresa.

La presente investigación se enfocará en definir un modelo de gestión para el proyecto CRTV en el cantón Cañar y así obtener un proyecto rentable y genere ingresos a corto plazo satisfaciendo la necesidad inmediata que solicita este cantón, teniendo en cuenta el beneficio ambientalista como la seguridad que tendrán todos los habitantes al momento de hacer uso del trasporte público así como de sus vehículos propios, el área de revacuno técnica vehicular sería un aporte fundamental para la reducción de accidentes causados por fallas técnicas y mecánicas que en esta área serán revisadas y aprobadas de manera que los vehículos estén aptos para el traslado de personas y su uso habitual.

El centro de revisión técnica vehicular se encargara de verificar aspectos tales como calibración de gases, alineación y balanceo, estado general de sistemas acoplados al vehículo, luces, sistemas de seguridad y aparatos de emergencia, de esta manera el departamento de matriculación tendrá una garantía más de seguridad para los automotores además de ello las personas de cantón no tendrán que recurrir al traslado a otras ciudades para obtener su matrícula ya que la incorporación de un centro de revisión técnica vehicular en el área de matriculación permite matricular vehículos comerciales y pesados además del beneficio económico también estaríamos completando la totalidad del servicio que brinda el área de matriculación y las fuentes de trabajo que implicaría la puesta en marcha de este departamento. 
Revista Interdisciplinaria de Humanidades, Educación, Ciencia y Tecnología

Año VII. Vol. VII. N¹2. Enero - Junio. 2021

Hecho el depósito de ley: pp201602FA4721

ISSN-L: 2542-3029; ISSN: 2610-802X

Universidad Nacional Experimental Francisco de Miranda (UNEFM). Santa Ana de Coro. Venezuela

Ángel Sebastián Martínez-Narváez; Juan Carlos Erazo-Álvarez; Cecilia Ivonne Narváez-Zurita Cristián Andrés Erazo-Álvarez

\section{Referencial Teórico}

\section{Definiciones de Estructura Organizacional Según Autores}

Es importante considerar dentro de los trabajos de investigación fuentes investigativas que sustente el documento y permitan conocer más sobre el tema, de este manera un punto importante a tratar es la estructura organizacional, la misma que se desarrolla dentro del estado de tiempo y lugar según varias condiciones en el sistema. Según /Minsal-Pérez \& Pérez-Rodríguez, 2007), indican que la estructura organizacional como un conjunto particular que mantienen en sus sistemas una distribución adecuada y un cierto orden en cada una de sus partes que la componen. (Vertice, 2008). La estructura es importante en el cumplimiento de los objetivos, estructura que sigue un proceso lógico mediante una dirección. En estas etapas se pueden describir algunas actividades que se pueden desarrollar a fin de alcanzar las metas u objetivos. Cada una de las personas deben hacer uso de los diferentes recursos o medios a fin de que mediante el sistema organizacional se pueda mejorar cada actividad y un mejor manejo de los recursos disponibles. Por otro lado, Robbins (2005), indica que

Una organización debe estar compuesta por varias personas que trabajan en virtud de cumplir cada proceso en la fijación de metas y objetivos de la empresa; Toda organización está compuesta por personas. Una persona que trabaja sola no es una organización y hacen faltan personas para realizar el trabajo, que se necesita para que las organizaciones cumplan sus metas.

Según la conceptualización se manifiesta que la unión hace la fuerza dentro de la organización necesitamos de todos en conjunto para poder alcanzar las metas. Según Merton (1968). "Una estructura social formal, racionalmente organizada, implica normas de actividad definidas con claridad en las que, idealmente cada serie de acciones esta funcionalmente relacionadas con los propósitos de la organización". De esta definición podemos decir que con una clara y bien definida estructura organizacional siguiendo las normas y procedimientos podemos alcanzar las metas de la organización. En este sentido, se tiene que "Una estructura organizacional es eficaz cuando facilita a las 


\section{CIENCIAMATRIA}

Revista Interdisciplinaria de Humanidades, Educación, Ciencia y Tecnología

Año VII. Vol. VII. N¹2. Enero - Junio. 2021

Hecho el depósito de ley: pp201602FA4721

ISSN-L: 2542-3029; ISSN: 2610-802X

Universidad Nacional Experimental Francisco de Miranda (UNEFM). Santa Ana de Coro. Venezuela

Ángel Sebastián Martínez-Narváez; Juan Carlos Erazo-Álvarez; Cecilia Ivonne Narváez-Zurita Cristián Andrés Erazo-Álvarez

personas la consecución de objetivos, metas y es eficiente cuando se consiguen con recursos o costos mínimos". (Chiavenato, 1999) Podemos decir que para la consecución de objetivos y metas de una organización es necesaria una estructura eficaz, para obtener resultados inmediatos con costos mínimos. Por otro lado, se tiene que "El conjunto de todas las formas de trabajo en diferentes tareas, y cómo se lleva a cabo la coordinación de estas". (Mintzberg, 2016) Podemos decir que para realizar cualquier tipo de trabajo dentro de la organización debemos seguir una serie de pasos bien definidos y así conseguir los objetivos propuestos.

Tipos de estructuras Organizacionales, Existen diversas tipologías y clasificaciones sobre las estructuras organizacionales .la mayoría de autores separan 4 tipos de estructuras: Estructura Simple Con un mínimo de personal dentro de las empresas "núcleos de personal" y "línea media", llevando la coordinación la cúspide estratégica mediante supervisión directa. Suelen darse en empresas pequeñas y de reciente creación (Publicaciones Vértice S.L., p. 10).

Dentro de la estructura organizacional esta la simple, que es caracterizada por niveles jerárquicos escasos, poca formalización, el trabajo se lo divide le forma vaga, es de tipo orgánica existiendo en cada etapa una supervisión para alcanzar la coordinación adecuada. Por ello en esta etapa es importante el director ejecutivo quien toma decisiones, así como controla cada funcionamiento dentro de la organización por lo que se convierte en el pilar fundamental de la estructura. (Psicologia Online, 2018). Estructura Burocrática: Las estructuras burocráticas tienen un cierto grado de normalización. Son más adecuadas para organizaciones más complejas y grandes. Este tipo de estructura esta dividida en dos como la burocracia maquinal y profesional, que poseen características similares pero para la parte esencial son muy diferenciadoras.

Tomando como la primera la Burocracia maquinal: es un término definido por Max Weber, en dónde establece la división en varias tareas que se las realiza rutinariamente, pero muy especializadas. En la parte nuclear de la estructura; para ello se genera medios 


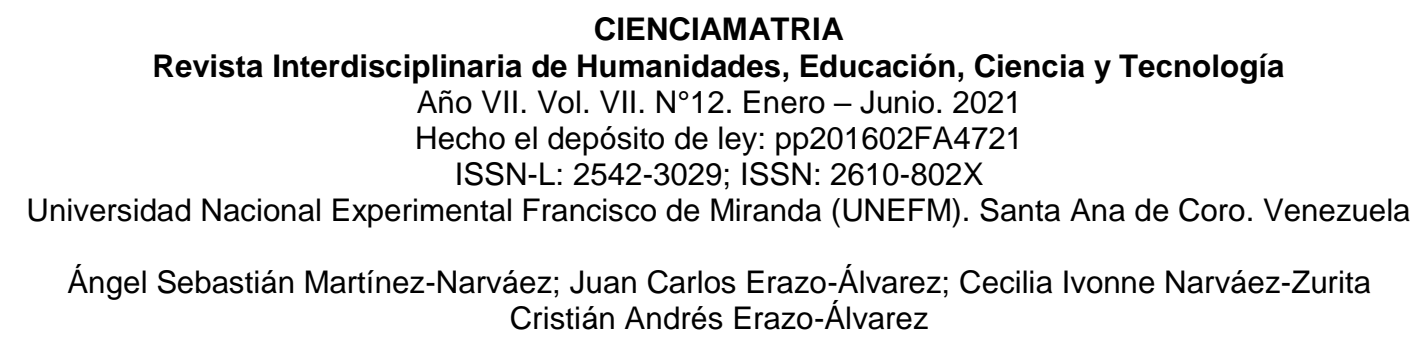

comunicativos que permitan que mediante normas a seguir, protocolos $u$ otros canales, de manera agrupada puedan priorizar interdependencias de proceso y de escala, siendo primordial esta etapa en la toma de decisiones con una clara distinción entre ápice, línea, núcleo y staffs.

En lo relacionado a la Burocracia profesional: esta tiene en su camino a diferentes entornos estables que por lo general son complejos, por lo que se la normalización de procesos no es un recurso, siendo necesario la normalización de habilidades, importante en la coordinación; descentralizando su incremento dirigiéndolo hacia el núcleo en dónde se realizan las operaciones. Como ejemplo se puede mencionar los establecimientos de salud como hospitales, las unidades educativas, empresas que producen de manera artesanal, y también las firmas de auditoría y contabilidad (Erazo \& Narváez, 2020).

Estructura Divisional izada: permite tomar decisiones de manera fácil a los encargados, considerando que la empresa crece día a día según el segmento de mercado, la venta de productos nuevos, inserción de la tecnología, así como nuevos procesos de tipo administrativo, etc. Es así que por estos cambios es importante que se tomen decisiones para acoplarse el nuevo entorno. (Mintzberg, 2016). Adhocracia: está fundamentado por el trabajo en equipo, en dónde se busca que el personal se adapte con procesos flexibles. Pero esta estructura evita que existan comportamientos formales, controlando que el trabajo sea dividido pero si orientado a una planificación oportuna y control de tareas. Según Mintzberg, menciona en lo referente a la Adhocracia Operativa: esta tienen como base principal la innovación, con el objetivo de resolver algunos problemas que tiene los clientes; formando un conjunto importante las tareas y operaciones en busca de un producto o servicio de calidad. Y como parte final se tiene la Adhocracia Administrativa: que está enfocado en el cumplimiento de cada etapa de la administración empresarial que brinda organización mejor funcionamiento. (Mintzberg, 2016). 
Universidad Nacional Experimental Francisco de Miranda (UNEFM). Santa Ana de Coro. Venezuela

Ángel Sebastián Martínez-Narváez; Juan Carlos Erazo-Álvarez; Cecilia Ivonne Narváez-Zurita Cristián Andrés Erazo-Álvarez

\section{Estructura Funcional}

En cuanto a la estructura formal, se tiene la siguiente definición:

La estructura organizacional vertical o funcional en donde las actividades están agrupadas en un trabajo ascendente, se identifica poca colaboración entre los departamentos funcionales al estar la organización dirigida y controlada por una jerarquía vertical en donde las decisiones están solo en las manos de los gerentes de nivel superior (Daft, 2011).

En este sentido, se comenta que la estructura funcional se basa en la jerarquía de la estructura controlada por el jefe da cada departamento en forma descendente esto dificulta a los jefes inmediatos a solucionar problemas con rapidez y celeridad debido a la poco o nula colaboración que existe entre departamento lo que genera que no se cumplan las metas propuestas en su inicio.

Por otro lado, se concibe la estructura funcional, como un "diseño que agrupa a las personas con base en su experiencia y pericia en común o porque utilizan los mismos recursos". (Gareth, 2013). acorde a Jones la estructura Funcional es un proyecto que brinda ventaja competitiva frente a sus competidores seleccionando al personal según sus habilidades y experiencias que tiene cada persona para sacar a relucir sus aptitudes y colocar en el lugar que le corresponde a cada uno de sus ellos según sus estudios, aptitudes y experiencias y así cumplir las metas propuestas, Según (Melinkoff, 1969), se evidencia que la estructura organizacional posee un bajo nivel de departamentalización, así como tramos largos de control, siendo una sola persona la autoridad realizándose escasos niveles de formalización, siendo flexible y contando con niveles verticales escasos. 
Revista Interdisciplinaria de Humanidades, Educación, Ciencia y Tecnología

Año VII. Vol. VII. N¹2. Enero - Junio. 2021

Hecho el depósito de ley: pp201602FA4721

ISSN-L: 2542-3029; ISSN: 2610-802X

Universidad Nacional Experimental Francisco de Miranda (UNEFM). Santa Ana de Coro. Venezuela

Ángel Sebastián Martínez-Narváez; Juan Carlos Erazo-Álvarez; Cecilia Ivonne Narváez-Zurita

Cristián Andrés Erazo-Álvarez

\section{Normativa Relativa a los Procesos de Implementación de un Centro de Revisión Vehicular}

Esta normativa establece según el directorio de la agencia nacional de regulación y control del transporte terrestre, transporte y seguridad vial, que en sus Artículo 52, que todas las personas están en total derecho de bienes o servicios que estén dentro de una óptima calidad, los mismos que pueden ser seleccionados con libertad, así también conocer su contenido sin ningún engaño. Por lo que la Asamblea Nacional en el año 2017, regido al numeral 25 del Artículo 66 describe el derecho a que las personas tienen a los diferentes bienes 0 a los servicios que ofertan diferentes empresas públicas 0 privadas, deben recibir buen trato, información veraz y oportuna.

Se refuerza este artículo con el numeral 27 en dónde se menciona el derecho de las personas que tienen a vivir en un ambiente totalmente sano, libre de contaminación ecológico y en armonía. Por lo que en el Artículo 266, manifiesta que todas las instituciones de tipo estatal, así como a todos quienes los involucran dentro de las mismas actuarán y se regirán a la función para la cual fueron designadas, según el Artículo 2 describen y dan prioridad de los derechos a la vida, mediante el cuidado del medio en donde vivimos para mejorar la calidad, derechos que se sustenta en la LOTTS y ANT; (ANT, 2015),

En base a los manifestado se establece que según el Registro 407 de manera oficial con la fecha del 31 del mes de diciembre del año 2014; que en su litera j) las competencias que se designan a las autoridades de los cantones están el autorizar e implementar los Centros de Revisión Técnica Vehicular, evaluando el estado mecánico, cada elemento de seguridad, ruido y emisión de gases. Que, el Art. 306 de la LOTTTSV de este reglamento expone que "Los propietarios de vehículos automotores están obligados a someter los mismos, a revisiones técnico mecánicas en los centros de revisión y control vehicular, autorizados conforme a la reglamentación que expida la Agencia Nacional de Tránsito". (p.41) (ANT, 2015). 
Según los registros del Artículo 307, mencionan que la revisión vehicular corresponden a los GADS, los mismos que tienen competencias, en dónde pueden revisar las condiciones técnicas y mecánicas de los vehículos, la seguridad, la comodidad y que cuenten con todos los implementos de revisión lo que garantiza su traslado y manejo por las diferentes carreteras del país. Cada uno de estos elementos que corresponden a la RTV podrán ser controlados por la ANT disposición que la sustenta en el Artículo 312, en dónde se menciona que todos los transportes de servicio tanto público o privado están en la obligación de ser revisados cada semestre y los otros vehículos una vez al año.

De esta manera se busca seguir con el cuidado de los choferes y vehículos para un servicio de calidad y seguridad; pero es de considerar aquellos vehículos que son nuevos no deben pasar por este procesos debido a que su kilometraje esta menor a los 1.000 kilómetros, según los respalda el Artículo 309 en dónde se establece la importancia que tiene el certificado de la revisión vehicular, por los que la ANT y los GADS deben estar completamente equipados de todos los materiales y recursos para otorgar la revisión y control de los vehículos, considerando que están sujetos cada entidad a fiscalización para un mejor control de los servicios que ofrecen a los choferes y vehículos dentro de cada cantón.

Siguiendo en la línea de Artículos que sustenta el tema de revisión vehicular, el Artículo 316, establece que cada una de las agencias de tránsito deben mantener una conminación constante con las unidades administrativas y GADS, por lo que los resultados obtenidos de las revisiones no podrán ser alterados, pero si es necesario cambios de parámetros estos deberán ser realizados bajo la autorización de la Agencia. (p.43)

En el caso de incumplimiento de cada parámetro y normas de tipo técnico establecidos por la ANT, deben ser cumplidas en su totalidad porque favorece a la seguridad de los conductores, evita el daño ambiental, por el control en la emisión de gases, también el control de los ruidos, pero para brindar un mejor servicio acorde a la demanda actual es 
importante hacer uso de la modernidad, así como la automatización, en los controles conjuntamente con la tecnificación, que permita una revisión de manera más simple y con garantía en el funcionamiento de cada automotor.

\section{Normativas NTE INEN para Revisión Técnica Vehicular.}

A continuación, se cita las normas técnicas INEN, referentes a la seguridad vial y contaminación por emisiones, que para el desarrollo de un centro de revisión técnica vehicular son necesarias. (NTE INEN 2 207:2002), Revisión Técnica Vehicular. Procedimientos. Se detalla los procedimientos que se deben seguir para realizar la revisión técnica vehicular obligatoria. Mediante el uso de sistemas computarizados y equipos.

\section{Proceso y Procedimiento de la Revisión Técnica Vehicular}

Los lugares en dónde se revisan los vehículos técnicamente tienen como meta el mejorar la condición que tiene el vehículo en la seguridad y del ambiente, con un proceso de inspección técnica de los sistemas mecánicos y elementos de seguridad de un vehículo, bajo parámetros nacionales suscritos por la Ley competente para dar como resultado de la inspección la autorización o no de la circulación del vehículo en el país. Es importante que se considera oportuna la revisión desde el punto mecánico de los automotores, en su seguridad, cada uno de los sistemas que lo componen, garantizando de esta manera la vida de las personas. (ANT RESOLUCION 070-DIR-2015, 2015).

En La inspección visual el encargado revisará cual es el estado de los elementos esenciales para que el vehículo esté en condiciones seguras para circular por las vías mediante observación, identifica ruidos, vibraciones anormales, holguras, puntos de corrosión, estado de las llantas, marcos de parabrisas, que las luces de retro, delanteras y traseras se encuentren funcionando etc. Este método se debe realizar según el tipo de vehículo según (NTE INEN 2 207:2002). 
Inspección mecatrónica: con el uso de aparatos e instrumentos mecatrónicas, electromecánicos y electrónicos revisarán el opacímetro, tacómetro, sonómetro, frenó metro y luxómetro. Opacímetro, para la prueba de emisión de humo, analizador de gases de escape, Sonómetro para niveles de ruido, Freno metro para comprobar el buen funcionamiento de frenos, Regloscopio para funcionamiento y alineación de luces, alineadora al paso y banco de suspensión.

Este tipo de inspección se realiza con software que se encuentra conectado a los equipos. Tipos de centros en dónde se procede con la revisión de vehículos. Se define como un espacio que se encargara de realizar las pruebas a cada automotor para mediante el la correcta revisión se pueda garantizar el funcionamiento emitiendo un comprobante que garantice las condiciones de buen funcionamiento de un vehículo entre mecánicas, la seguridad y cuidados ambientales. Cada centro que se encarga de la revisión pueden ser clasificados de dos maneras en:

\section{Centro de Revisión Fijo}

El centro de revisión fijo es un espacio con gran infraestructura y son implementados cuando una región supera su parque automotor los 15.000 vehículos, deben estar con disponibilidad de espacios como parqueaderos, con techo y cerrado debe mantener una línea correcta de orientación, buena iluminación, ventilación, acústico y aireación lo que permite que le revisión sea óptima, así también debe contar con los servicios básicos, sistemas de vías y señalización adecuadas para un mejor servicio en ingreso y salida y sobre todo con recursos humanos como inspector, recepción, personal guardianía y entrega de documentación. (ANT RESOLUCION 070DIR-2015, 2015)

\section{Centro de Revisión Móvil}

Tiene la capacidad de moverse para que se cambie de lugar y pueda ofrecer su servicio. Este tipo de centro cuenta con los mismos recursos que los de un centro fijo y también 
está encargado de emitir un comprobante para garantizar las condiciones de buen funcionamiento del vehículo. Para la movilización estos centros deben contar con un remolque así también el uso de líneas longitudinales y transversales pudiendo hacer la revisión en vehículos tanto pesados como livianos. (ANT RESOLUCION 070-DIR-2015, 2015). Este tipo de centro puede operar en cualquier parte, pero debe permitir una libre circulación de vehículos, además cuentan con servicios básicos y la superficie donde funcionara debe ser estable y plana. (ANT RESOLUCION 070-DIR-2015, 2015)

Tipos de líneas

Cada centro que se encarga de la revisión tienen líneas utilizadas de acuerdo al tipo de automotor, tomando en consideración el área de trabajo o infraestructura; contando con personal para revisar los vehículos así como el control de los mismos; Según la clase de vehículos que se deben inspeccionar, se clasifican en los siguientes tipos: Línea de Inspección Tipo Menor La línea tipo menor está diseñada para la revisión de vehículos menores, tales como motocicletas, tricótomos, moto taxis, moto furgones, etc. (N34222004, Resolución Directorial, 2004). Línea de Inspección Tipo Liviano está destinada a la revisión de los vehículos livianos que según la clasificación vehicular están comprendidos los automóviles sedan, station wagon, camionetas, remolques, etc., con un peso máximo de hasta 3500 Kg. (N3422-2004, Resolución Directorial, 2004).

Se encarga la línea pesada de la revisión de camiones, también buses, remolques o semirremolques los mismos que se encuentran dentro de un peso de neto superior a los 3500 Kg. (N3422-2004, Resolución Directorial, 2004). Línea de Inspección Tipo Mixta La línea tipo mixta está diseñada para realizar la inspección de vehículos de tipo liviano y pesado. A nivel de Latinoamérica los Modelos de inspección técnica vehicular han sido implementados buscando ayuda de inversionistas o de las autoridades competentes a fin de facilitar espacios para la revisión de tipo técnica de los vehículos. 
Revista Interdisciplinaria de Humanidades, Educación, Ciencia y Tecnología

Año VII. Vol. VII. N¹2. Enero - Junio. 2021

Hecho el depósito de ley: pp201602FA4721

ISSN-L: 2542-3029; ISSN: 2610-802X

Universidad Nacional Experimental Francisco de Miranda (UNEFM). Santa Ana de Coro. Venezuela

Ángel Sebastián Martínez-Narváez; Juan Carlos Erazo-Álvarez; Cecilia Ivonne Narváez-Zurita Cristián Andrés Erazo-Álvarez

\section{MÉTODO}

La investigación tuvo primordialmente un alcance metodológico de tipo descriptivo con diseño no experimental transversal, realizada en una muestra poblacional de 89 que poseen un vehículo automotor y están sujetas a matricular y realizar revisión técnica vehicular en el cantón Cañar - Ecuador. Siendo encuestados de modo online a través de un cuestionario en escala de Likert de cinco alternativas de respuestas, siendo validado por juicio de expertos y cálculo de fiabilidad de Alfa de Cronbach con resultado de 0,89 siendo calificado como apto para ser aplicado, mientras que la información recopilada fue procesada a través de la estadística descriptiva.

\section{RESULTADOS}

La presente investigación toma como base los resultados de las encuestas realizadas a la ciudadanía del cantón cañar, para la creación de un centro de revisión técnica vehicular, en cuanto a la presentación de los resultados han sido resumidos en dimensiones.

\section{Dimensión: importancia de la gestión del departamento de matriculación}

Para este aspecto el $41,7 \%$ de los encuestados han contestado que la gestión del departamento de matriculación es regular, el $50 \%$ de las personas propietarios de vehículos han contestado que es buena y el $8,3 \%$ de encuestados han contestado que la gestión del este departamento es mala.

\section{Dimensión: beneficio social}

Para la siguiente dimensión las personas encuestadas el $41,7 \%$ de las mismas no saben cuál es el beneficio social que tiene la incorporación del departamento de revisión técnica vehicular, y el 58,3 de personas encuestadas restantes contestaros que si conocen el beneficio de incorporar este centro en el cantón cañar. 


\section{Dimensión: contaminación}

En este apartado los encuestados contestaron el 43,8\% que la emisión por gases contaminantes en el cantón cañar es alta, el 52,2 contestaron que es media, y el 2,1\% replicaron que es baja.

\section{Dimensión: seguridad en el transporte publico}

En este ítem la ciudadanía del cantón cañar contesto con el $64,6 \%$ que es media, el $18,8 \%$ corresponde a las personas que contestaron que esa seguridad es media y, el 16,7\% son las personas que escogieron por la opción de baja.

\section{PROPUESTA}

En el modelo de gestión, es de considerar un punto importante para su aplicación como una herramienta de revisión en la parte técnica del automotor; que nos permita ejecutar de mejor manera este proceso y garantizar la disminución de gases contaminantes de vehículos, particulares, comerciales, pesados y motos y así garantizando la sostenibilidad de este proyecto.

\section{Esquema de la propuesta del Modelo de Gestión dirigida al CRTV en el cantón Cañar.}

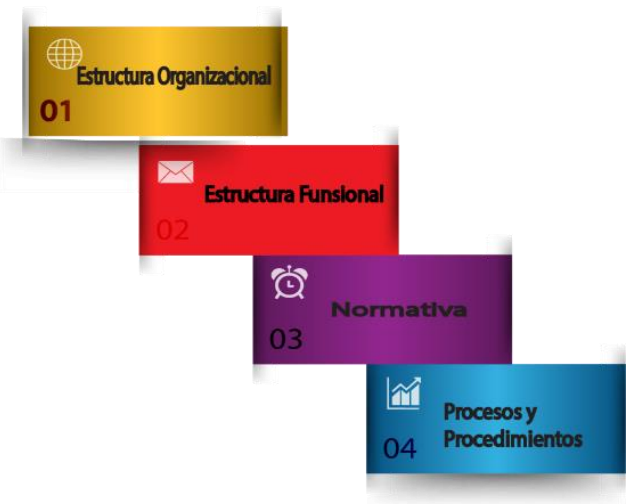

Figura 1. Esquema de la Propuesta para la incorporación del CRTV del Cantón Cañar 


\section{CIENCIAMATRIA}

Revista Interdisciplinaria de Humanidades, Educación, Ciencia y Tecnología

Año VII. Vol. VII. N¹2. Enero - Junio. 2021

Hecho el depósito de ley: pp201602FA4721

ISSN-L: 2542-3029; ISSN: 2610-802X

Universidad Nacional Experimental Francisco de Miranda (UNEFM). Santa Ana de Coro. Venezuela

Ángel Sebastián Martínez-Narváez; Juan Carlos Erazo-Álvarez; Cecilia Ivonne Narváez-Zurita Cristián Andrés Erazo-Álvarez

\section{Estructura Organizacional}

A continuación presentamos la estructura organización de acuerdo a los lineamientos que exige este centro de revisión el mismo que consta de una estructura jerárquica como observamos en la siguiente figura.

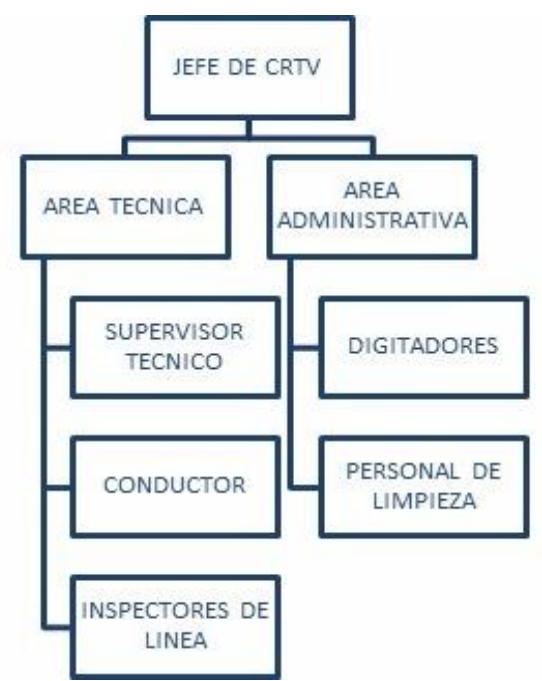

Figura 2. Estructura Organizacional para el CRTV.

\section{Estructura Funcional}

A continuación presentamos las actividades que van a realizar cada uno de los empleados que constituyen el organigrama. 
CIENCIAMATRIA

Revista Interdisciplinaria de Humanidades, Educación, Ciencia y Tecnología

Año VII. Vol. VII. N¹2. Enero - Junio. 2021

Hecho el depósito de ley: pp201602FA4721

ISSN-L: 2542-3029; ISSN: 2610-802X

Universidad Nacional Experimental Francisco de Miranda (UNEFM). Santa Ana de Coro. Venezuela

Ángel Sebastián Martínez-Narváez; Juan Carlos Erazo-Álvarez; Cecilia Ivonne Narváez-Zurita

Cristián Andrés Erazo-Álvarez

\section{Tabla 1.}

Esquema Funcional del CRTV del Cantón Cañar.

\begin{tabular}{|l|l|}
\hline CARGO & TAREA/REQUERIMIENTO \\
\hline Director Operativo & $\begin{array}{l}\text { Proceder con la administración del CRTV en los diferentes } \\
\text { procesos como el administrativo y técnico que son los } \\
\text { responsables del buen funcionamiento en el recursos de } \\
\text { tipo humano. }\end{array}$ \\
\hline Supervisor Técnico & $\begin{array}{l}\text { Se debe mejorar la organización mediante el trabajo de } \\
\text { inspección para los conductores en cada uno de los } \\
\text { procesos de en le revisión logrando brindar veracidad y } \\
\text { confiabilidad. Esto basado en tareas de mantenimiento de } \\
\text { cada equipo asi como mejorando y actualizando los } \\
\text { conocimientos del personal a cargo. } \\
\text { Asi tambien es importante la reportación de informes sobre } \\
\text { las novedades encontradas estas por lo general cabe la } \\
\text { responsabilidad en un profesionales en el área de Mecánica } \\
\text { Automoríz. }\end{array}$ \\
\hline Digitadores & $\begin{array}{l}\text { Son quienes tienen la licencia profesional y pueden manejar } \\
\text { sus vehículos según la líena de revisión para } \\
\text { posteriormente ser aprobados. }\end{array}$ \\
\hline Conductores & $\begin{array}{l}\text { Es de considerar que esta persona bene tener } \\
\text { conocimientos importantes en la parte técnica, que le } \\
\text { permita llevar cada proceso de revisión asi como su } \\
\text { verificación tecnica según la normativa que se encuentra } \\
\text { vigente. }\end{array}$ \\
\hline $\begin{array}{l}\text { Estan encargados de revisar los documentos y que los } \\
\text { mismos tenda validez y letigimidad, por parte de los }\end{array}$ \\
\hline Inspectores de Línea
\end{tabular}


CIENCIAMATRIA

Revista Interdisciplinaria de Humanidades, Educación, Ciencia y Tecnología

Año VII. Vol. VII. N¹2. Enero - Junio. 2021

Hecho el depósito de ley: pp201602FA4721

ISSN-L: 2542-3029; ISSN: 2610-802X

Universidad Nacional Experimental Francisco de Miranda (UNEFM). Santa Ana de Coro. Venezuela

Ángel Sebastián Martínez-Narváez; Juan Carlos Erazo-Álvarez; Cecilia Ivonne Narváez-Zurita Cristián Andrés Erazo-Álvarez

\begin{tabular}{|l|l|}
\hline & $\begin{array}{l}\text { conductores, para que cuando se pueda terminar con el } \\
\text { proceso emitan certificados, regidos a conocimiento en el } \\
\text { área legislativa y computación. }\end{array}$ \\
\hline Personal de Limpieza & $\begin{array}{l}\text { Debe mantener todo el espacio de trabajo limpio, asi como } \\
\text { el mantenimiento de las diferentes áreas de revisión. }\end{array}$ \\
\hline
\end{tabular}

\section{Normativa}

Según esta normativa regida a la NTE INEN 2349 (2003). Revisión Técnica vehicular los CRTV en cada una sus líneas deben contener el siguiente equipamiento con las especificaciones que nos muestran las siguientes tablas:

a) Banco de pruebas para deriva dinámica (Side Slip Tester), en base a las características detalladas:

\section{Tabla 2.}

Banco de Pruebas para deriva dinámica.

\begin{tabular}{|l|l|}
\hline \multicolumn{1}{|c|}{ PARAMETRO } & \multicolumn{1}{c|}{ REQUERIMIENTO } \\
\hline Tipo & $\begin{array}{l}\text { Debe ser automática de metal, que debe estar } \\
\text { empotrada en el piso y que se pueda deslizar. }\end{array}$ \\
\hline Rango mínimo de medición & De $-15 \mathrm{a}+15 \mathrm{~m} . \mathrm{km}-1$ \\
\hline Velocidad aproximada de paso & $4 \mathrm{~km} \cdot \mathrm{h}-1$ \\
\hline Capacidad mínima portante & $\begin{array}{l}1500 \mathrm{~kg} \text { en el caso de vehñiculos considerados como } \\
\text { livianos } \\
8000 \mathrm{~kg} \text { para los automotores pesados }\end{array}$ \\
\hline $\begin{array}{l}\text { Valor de una división de escala } \\
\text { (resolución) }\end{array}$ & \multicolumn{1}{|c|}{$\mathrm{m} \cdot \mathrm{km} \mathrm{-1}$} \\
\hline
\end{tabular}

b) Para las suspensiones, las preguntas deben estar dentro de las calificaciones de medición automática de la delantera y posterior en su eficiencia, la amplitud que 
CIENCIAMATRIA

Revista Interdisciplinaria de Humanidades, Educación, Ciencia y Tecnología

Año VII. Vol. VII. N¹2. Enero - Junio. 2021

Hecho el depósito de ley: pp201602FA4721

ISSN-L: 2542-3029; ISSN: 2610-802X

Universidad Nacional Experimental Francisco de Miranda (UNEFM). Santa Ana de Coro. Venezuela

Ángel Sebastián Martínez-Narváez; Juan Carlos Erazo-Álvarez; Cecilia Ivonne Narváez-Zurita Cristián Andrés Erazo-Álvarez

poses asi como la oscilación y si existe resonancia en las ruedas, por lo que se sugiere tomar en cuenta los siguientes indicadores:

\section{Tabla 3.}

Banco de pruebas para suspensiones.

\begin{tabular}{|l|l|}
\hline PARAMETRO & REQUERIMIENTO \\
\hline Tipo & $\begin{array}{l}\text { Deb estar al ras del piso en primer lugar, } \\
\text { doble y oscilante en su amplitud y la } \\
\text { frecuencia de la misma determinando } \\
\text { variables automáticas. }\end{array}$ \\
\hline Ancho de vía del vehículo & $850 \mathrm{~mm}$ como medida interna como mínimo \\
& $2000 \mathrm{~mm}$ a lo máximo que se llega en lo \\
& externo \\
\hline Capacidad portante mínima & $1500 \mathrm{~kg}$ por eje \\
\hline Valor de una división de escala (resolución) & $1 \%$ en la eficiencia; $1 \mathrm{~mm}$ en la amplitud \\
\hline
\end{tabular}

c) Banco de pruebas para frenos, facilitan el control de forma automática el porcentajes el frenado, (servicio y parqueo), también se considerar la existencia de desequilibrio dinámico cuando se frena esto también medido en porcentajes, en cuanto a los tambores se controla la ovalización, importante el control de pandeo entre los discos con ello la fuerza con la que frenan cada rueda así como también esta pruebas están dirigidas para los vehículos con el sistema de antibloqueo, el sistema que tienen transmisión permanente estos en las cuatro ruedas, los mismos que cuenta con caja de velocidades de tipo manual, y otras automáticas y semiautomáticas; estos vehículos deben contar con las características técnicas que se mencionan a continuación. 
CIENCIAMATRIA

Revista Interdisciplinaria de Humanidades, Educación, Ciencia y Tecnología

Año VII. Vol. VII. N¹2. Enero - Junio. 2021

Hecho el depósito de ley: pp201602FA4721

ISSN-L: 2542-3029; ISSN: 2610-802X

Universidad Nacional Experimental Francisco de Miranda (UNEFM). Santa Ana de Coro. Venezuela

Ángel Sebastián Martínez-Narváez; Juan Carlos Erazo-Álvarez; Cecilia Ivonne Narváez-Zurita Cristián Andrés Erazo-Álvarez

\section{Tabla 4.}

Banco de pruebas para frenos.

\begin{tabular}{|l|l|}
\hline PARAMETRO & REQUERIMIENTO \\
\hline Tipo de Frenómetro & $\begin{array}{l}\text { Se lo realiza por eje uno por uno, la base } \\
\text { debe ser antideslizante, firme a ras del } \\
\text { piso. }\end{array}$ \\
\hline Coeficiente mínimo de fricción $(\mu)$ & $\begin{array}{l}0,8 \text { se lo evalua en dos estados seco o en } \\
\text { mojado }\end{array}$ \\
\hline Carga mínima de absorción sobre rodillos & $\begin{array}{l}3.000 \text { kg estos en automotores } \\
\text { considerados como livianos } \\
7.500 \text { kg para automotores pesados }\end{array}$ \\
\hline Valor de una división de escala (resolución) & $\begin{array}{l}1 \% \text { evaluado en la eficiencia asi como en } \\
\text { su equilibrio; } 0,1 \text { daN con que tipo de } \\
\text { fuerza aplica cuando se frena. }\end{array}$ \\
\hline Dispositivos de seguridad & $\begin{array}{l}\text { Para el bloqueo de las ruedas debe ser } \\
\text { automático. } \\
\text { Al iniciar en cero debe estar automático } \\
\text { para evaluar antes de la prueba. }\end{array}$ \\
\hline
\end{tabular}

ABS: Antilock Breaking System (Sistema Antibloqueo de Frenos).

d) Se aplica en plantas fijas un monitoreo mediante el sistema automático.

e) Torre de inflado de llantas, con manómetro incorporado, que permita la determinación de la presión en la cámara del neumático con una resolución de 3,45 Pa (0,5 psi).

f) Dispositivo automático de pesaje del vehículo, en línea con los sistemas de pruebas de frenos y suspensiones. Este equipo puede estar incorporado al banco de pruebas de suspensiones o de frenado.

g) Detector de profundidad de labrado de neumáticos, con una resolución de 0,1 mm. 


\section{CIENCIAMATRIA}

Revista Interdisciplinaria de Humanidades, Educación, Ciencia y Tecnología

Año VII. Vol. VII. N¹2. Enero - Junio. 2021

Hecho el depósito de ley: pp201602FA4721

ISSN-L: 2542-3029; ISSN: 2610-802X

Universidad Nacional Experimental Francisco de Miranda (UNEFM). Santa Ana de Coro. Venezuela

Ángel Sebastián Martínez-Narváez; Juan Carlos Erazo-Álvarez; Cecilia Ivonne Narváez-Zurita Cristián Andrés Erazo-Álvarez

h) Luxómetro con regloscopio autoalimente de eje vertical y horizontal, con las siguientes características técnicas:

\section{Tabla 5.}

Luxómetro parámetros.

\begin{tabular}{|l|l|}
\hline PARÁMETRO & REQUERIMIENTO \\
\hline Rango de medición & $\begin{array}{l}\text { De } 0 \text { a mínimo } 250000 \text { candelas } \\
(2,69 \times 106 \text { lux })\end{array}$ \\
\hline Alineación con el eje del vehículo & Automática \\
\hline
\end{tabular}

i) Debe el banco detector de holguras, estar empotrado en una fosa que esta iluminada o también puede ser un elevador cumpliendo con lo siguiente:

\section{Tabla 6.}

Banco detector de holguras.

\begin{tabular}{|l|l|}
\hline \multicolumn{1}{|c|}{ PARÁMETRO } & \multicolumn{1}{|c|}{ REQUERIMIENTO } \\
\hline Tipo de banco & $\begin{array}{l}\text { Deb esta compuestos por 2 placas, estas mediante el } \\
\text { uso de movimientos como el longitudinal, } \\
\text { incorporando a ello los transversales, de forma } \\
\text { igualitaria asi como en lo contario. Debe estar } \\
\text { empotrada en el piso o pavimento en una fosa o caso } \\
\text { contrario puede hacers uso de un elevador. }\end{array}$ \\
\hline Capacidad portante & $\begin{array}{l}1000 \mathrm{~kg} \text { determinando la placa y si es vehículo } \\
\text { liviano. } \\
3500 \mathrm{~kg} \text { De la misma manera por la placa y si es un } \\
\text { automotor pesado. }\end{array}$ \\
\hline $\begin{array}{l}\text { lluminación para detección } \\
\text { visual }\end{array}$ & $\begin{array}{l}\text { Se hace uso de una lámpara de tipo alógena que sea } \\
\text { por lo general regulable y con una bueno potencia. }\end{array}$ \\
\hline
\end{tabular}


CIENCIAMATRIA

Revista Interdisciplinaria de Humanidades, Educación, Ciencia y Tecnología

Año VII. Vol. VII. N¹2. Enero - Junio. 2021

Hecho el depósito de ley: pp201602FA4721

ISSN-L: 2542-3029; ISSN: 2610-802X

Universidad Nacional Experimental Francisco de Miranda (UNEFM). Santa Ana de Coro. Venezuela

Ángel Sebastián Martínez-Narváez; Juan Carlos Erazo-Álvarez; Cecilia Ivonne Narváez-Zurita Cristián Andrés Erazo-Álvarez

j) Analizador de gases: Se procede con el analizador que establece 4 gases que tiene la posibilidad de actualizar hasta 5 gases con el canal conocido como NOx, que tienen las características que se mencionan a continuación:

Tabla 7.

Analizador de gases.

\begin{tabular}{|c|c|c|}
\hline PARÁMETRO & \multicolumn{2}{|l|}{ REQUERIMIENTO } \\
\hline Características generales & \multicolumn{2}{|c|}{$\begin{array}{l}\text { Mide de forma general y reporta de forma automática que } \\
\text { volumen de concentración existe de } \mathrm{CO}, \mathrm{CO} 2, \mathrm{HC} \text { '2, que } \\
\text { emiten los vehículos por el escape, que por lo general son } \\
\text { de } 4 \text { cilindros, a gasolina, el GLP o GNC, se basan al } \\
\text { cumplimiento Internacional con la certificación del } \\
\text { fabricante. }\end{array}$} \\
\hline $\begin{array}{l}\text { Especificaciones } \\
\text { adicionales }\end{array}$ & \multicolumn{2}{|c|}{$\begin{array}{l}\text { Dentro de las especificaciones se debe adicionar con qué } \\
\text { velocidad gira el motor en RPM, que es una operación } \\
\text { mediante el cálculo con una fórmula conocida como Bret } \\
\text { Shneider asi como calcula la temperatura con la que se } \\
\text { encuentra el aceite. }\end{array}$} \\
\hline \multirow[t]{7}{*}{ Rangos de medición } & Se determina cual es la variables & $\begin{array}{l}\text { Es evaluado por el } \\
\text { rango en la que se } \\
\text { mide }\end{array}$ \\
\hline & (CO) & $0-10 \%$ \\
\hline & (CO2) & $0-16 \%$ \\
\hline & $(\mathrm{O} 2)$ & $0-21 \%$ \\
\hline & No Hidrocarburos & $0-5000 \mathrm{ppm}$ \\
\hline & De tipo combustionados & \\
\hline & $\begin{array}{l}\text { La velocidad con la que tiene o } \\
\text { realiza el giro el motor }\end{array}$ & $0-10000 \mathrm{rpm}$ \\
\hline
\end{tabular}


CIENCIAMATRIA

Revista Interdisciplinaria de Humanidades, Educación, Ciencia y Tecnología

Año VII. Vol. VII. N¹2. Enero - Junio. 2021

Hecho el depósito de ley: pp201602FA4721

ISSN-L: 2542-3029; ISSN: 2610-802X

Universidad Nacional Experimental Francisco de Miranda (UNEFM). Santa Ana de Coro. Venezuela

Ángel Sebastián Martínez-Narváez; Juan Carlos Erazo-Álvarez; Cecilia Ivonne Narváez-Zurita Cristián Andrés Erazo-Álvarez

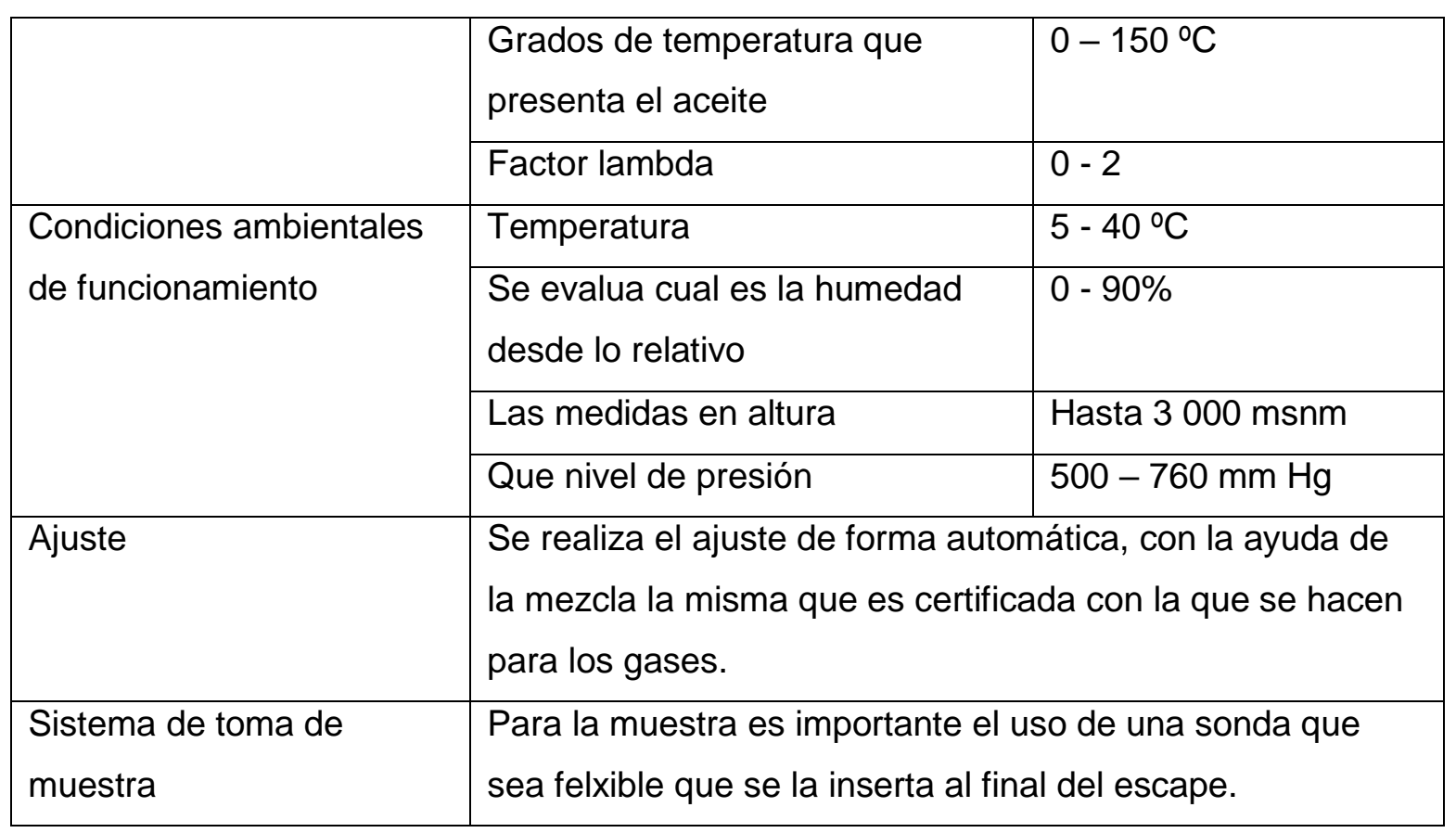

\section{Procesos y Procedimientos}

Detalle de áreas de líneas de revisión

La siguiente tabla nos indica las áreas mínimas que debe tener los CRTV de acuerdo con el número de líneas previstas para la misma:

\section{Tabla 8.}

Áreas mínimas CRTV.

\begin{tabular}{|l|l|l|l|l|l|}
\hline Cantidad de líneas & 1 & 2 & 3 & 4 & 5 \\
\hline $\begin{array}{l}\text { Medidas en superficie de los terrenos (m2) } \\
\text { mínimo }\end{array}$ & 2000 & 3000 & 4000 & 5000 & 6000 \\
\hline $\begin{array}{l}\text { Medidas en superficie que es la nave en lo } \\
\text { referente a revisión (m²) mínimo }\end{array}$ & 112 & 212 & 312 & 412 & 512 \\
\hline $\begin{array}{l}\text { Medidas de la superficie en los contextos de } \\
\text { servicios o zonas }\left(\mathrm{m}^{2}\right) \text { mínimo }\end{array}$ & 80 & 100 & 120 & 140 & 160 \\
\hline
\end{tabular}


CIENCIAMATRIA

Revista Interdisciplinaria de Humanidades, Educación, Ciencia y Tecnología

Año VII. Vol. VII. N¹2. Enero - Junio. 2021

Hecho el depósito de ley: pp201602FA4721

ISSN-L: 2542-3029; ISSN: 2610-802X

Universidad Nacional Experimental Francisco de Miranda (UNEFM). Santa Ana de Coro. Venezuela

Ángel Sebastián Martínez-Narváez; Juan Carlos Erazo-Álvarez; Cecilia Ivonne Narváez-Zurita Cristián Andrés Erazo-Álvarez

\section{CARGAS ELÉCTRICAS REFERENCIALES DE LOS EQUIPOS.}

Este dimensionamiento se ha realizado en función a equipamiento existente en algunas líneas de revisión técnica de provincias aledañas, sin embargo, el criterio de selección está acorde a un estándar alto de demanda energética para dar cabida a múltiples ofertantes que puedan a futuro equipar las líneas del presente estudio.

\section{Tabla 9.}

Cargas Eléctricas.

\begin{tabular}{|c|c|c|}
\hline EQUIPO & CONSUMO & CONEXIÓN \\
\hline Frenómetro Livianos & $2 \times 4,7 \mathrm{KW}$ & $3 \times 220 V+N+T$ \\
\hline Frenómetro Pesados & $2 \times 11 \mathrm{KW}$ & $3 \times 220 V+N+T$ \\
\hline Bco. Suspensiones & $2 \times 3 \mathrm{KW}$ & $1 \times 230 V+N+T$ \\
\hline Detector de Holguras livianos & 2,2 KW & $3 \times 220 V+N+T$ \\
\hline Detector de Holguras pesados & $3 \mathrm{KW}$ & $3 \times 220 V+N+T$ \\
\hline Analizador de Gases & $0.1 \mathrm{KW}$ & $1 \times 110-220 \mathrm{~V}+\mathrm{N}+\mathrm{T}$ \\
\hline Opacímetro & $0.1 \mathrm{~kW}$ & $1 \times 110-220 V+N+T$ \\
\hline Luxómetro & Baterías & $1 \times 110 V+N+T$ \\
\hline Velocímetro Livianos & $3 \mathrm{KW}$ & $3 \times 220 \mathrm{~V}+\mathrm{N}+\mathrm{T}$ \\
\hline Velocímetro Pesados & $3 \mathrm{KW}$ (hidráulico) & $3 \times 220 V+N+T$ \\
\hline Frenómetro motocicletas & $2.5 \mathrm{KW}$ & $3 \times 220 V+N+T$ \\
\hline Regloscopio motocicletas & $0.1 \mathrm{~kW}$ & $1 \times 110 V+N+T$ \\
\hline \begin{tabular}{|lll} 
Analizador & de & gases \\
motocicletas & & \\
\end{tabular} & $0.1 \mathrm{~kW}$ & $1 \times 110-220 \mathrm{~V}+\mathrm{N}+\mathrm{T}$ \\
\hline
\end{tabular}


CIENCIAMATRIA

Revista Interdisciplinaria de Humanidades, Educación, Ciencia y Tecnología

Año VII. Vol. VII. N¹2. Enero - Junio. 2021

Hecho el depósito de ley: pp201602FA4721

ISSN-L: 2542-3029; ISSN: 2610-802X

Universidad Nacional Experimental Francisco de Miranda (UNEFM). Santa Ana de Coro. Venezuela

Ángel Sebastián Martínez-Narváez; Juan Carlos Erazo-Álvarez; Cecilia Ivonne Narváez-Zurita Cristián Andrés Erazo-Álvarez

\begin{tabular}{|l|l|l|}
\hline Compresor & $7.5 \mathrm{KW}$ & $3 \times 220 \mathrm{~V}+\mathrm{N}+\mathrm{T}$ \\
\hline
\end{tabular}

\section{Cargas eléctricas de los equipos de revisión}

\section{Tabla 10.}

Tablas eléctricas y consumo.

\begin{tabular}{|l|l|l|}
\hline \multicolumn{1}{|c|}{ EQUIPO } & \multicolumn{1}{|c|}{ CONSUMO } & \multicolumn{1}{c|}{ CONEXIÓN } \\
\hline Frenómetro Livianos & $2 \times 4,7 \mathrm{KW}$ & $3 \times 220 \mathrm{~V}+\mathrm{N}+\mathrm{T}$ \\
\hline Bco. Suspensiones & $2 \times 3 \mathrm{KW}$ & $1 \times 230 \mathrm{~V}+\mathrm{N}+\mathrm{T}$ \\
\hline Detector de Holguras livianos & $2,2 \mathrm{KW}$ & $3 \times 220 \mathrm{~V}+\mathrm{N}+\mathrm{T}$ \\
\hline Analizador de Gases & $0.1 \mathrm{KW}$ & $1 \times 110-220 \mathrm{~V}+\mathrm{N}+\mathrm{T}$ \\
\hline Opacímetro & $0.1 \mathrm{KW}$ & $1 \times 110-220 \mathrm{~V}+\mathrm{N}+\mathrm{T}$ \\
\hline Luxómetro & Baterías & $1 \times 110 \mathrm{~V}+\mathrm{N}+\mathrm{T}$ \\
\hline Velocímetro Livianos & $3 \mathrm{KW}$ & $3 \times 220 \mathrm{~V}+\mathrm{N}+\mathrm{T}$ \\
\hline TOTAL & $21 \mathrm{KW}$ & \multicolumn{1}{|c|}{} \\
\cline { 2 - 3 } & &
\end{tabular}

\section{Cargas eléctricas de los equipos de revisión línea livianos}

\section{Tabla 11.}

Tablas eléctricas y consumo.

\begin{tabular}{|l|l|l|}
\hline QUIPO & CONSUMO & CONEXIÓN \\
\hline Frenómetro Pesados & $2 \times 11 \mathrm{KW}$ & $3 \times 220 \mathrm{~V}+\mathrm{N}+\mathrm{T}$ \\
\hline Bco. Suspensiones & $2 \times 3 \mathrm{KW}$ & $1 \times 230 \mathrm{~V}+\mathrm{N}+\mathrm{T}$ \\
\hline Detector de Holguras pesados & $3 \mathrm{KW}$ & $3 \times 220 \mathrm{~V}+\mathrm{N}+\mathrm{T}$ \\
\hline Opacímetro & $0.1 \mathrm{KW}$ & $1 \times 110-220 \mathrm{~V}+\mathrm{N}+\mathrm{T}$ \\
\hline Luxómetro & Baterías & $1 \times 110 \mathrm{~V}+\mathrm{N}+\mathrm{T}$ \\
\hline Velocímetro Pesados & $3 \mathrm{KW}$ (hidráulico) & $3 \times 220 \mathrm{~V}+\mathrm{N}+\mathrm{T}$ \\
\hline
\end{tabular}




\section{CIENCIAMATRIA}

Revista Interdisciplinaria de Humanidades, Educación, Ciencia y Tecnología

Año VII. Vol. VII. N¹2. Enero - Junio. 2021

Hecho el depósito de ley: pp201602FA4721

ISSN-L: 2542-3029; ISSN: 2610-802X

Universidad Nacional Experimental Francisco de Miranda (UNEFM). Santa Ana de Coro. Venezuela

Ángel Sebastián Martínez-Narváez; Juan Carlos Erazo-Álvarez; Cecilia Ivonne Narváez-Zurita Cristián Andrés Erazo-Álvarez

\section{TOTAL}

\section{$35 \mathrm{KW}$}

\section{Cargas eléctricas de los equipos de revisión línea pesados}

\section{Tabla 12.}

Tablas eléctricas y consumo.

\begin{tabular}{|c|c|c|}
\hline EQUIPO & CONSUMO & CONEXIÓN \\
\hline Bco. Suspensiones & $2 \times 3 \mathrm{KW}$ & $1 \times 230 V+N+T$ \\
\hline Analizador de Gases & $0.1 \mathrm{KW}$ & $1 \times 110-220 \mathrm{~V}+\mathrm{N}+\mathrm{T}$ \\
\hline Velocímetro Livianos & $3 \mathrm{KW}$ & $3 \times 220 V+N+T$ \\
\hline Frenó metro motocicletas & $2.5 \mathrm{KW}$ & $3 \times 220 V+N+T$ \\
\hline Regloscopio motocicletas & $0.1 \mathrm{KW}$ & $1 \times 110 V+N+T$ \\
\hline $\begin{array}{l}\text { Analizador } \quad \text { de } \\
\text { motocicletas }\end{array}$ & gases $0.1 \mathrm{KW}$ & $1 \times 110-220 \mathrm{~V}+\mathrm{N}+\mathrm{T}$ \\
\hline TOTAL & $12 \mathrm{KW}$ & \\
\hline
\end{tabular}

Cargas eléctricas de los equipos de revisión línea motocicletas

\section{Tabla 13.}

Tablas eléctricas y consumo.

\begin{tabular}{|l|l|}
\hline LINEAS & CONSUMO \\
\hline $\begin{array}{l}\text { Se establece } 3 \text { líneas que son usadas en la revisión de } \\
\text { automotores de tipo livianos. }\end{array}$ & $63 \mathrm{KW}$ \\
\hline $\begin{array}{l}\text { Y se establece } 2 \text { líneas que son usadas en la revisión de } \\
\text { vehículos que son definidos como pesados. }\end{array}$ & $70 \mathrm{KW}$ \\
\hline 1 líneas para revisión de motocicletas & 12 \\
\hline Compresor & $7.5 \mathrm{KW}$ \\
\hline TOTAL & $152.5 \mathrm{KW}$ \\
\hline
\end{tabular}


Cargas eléctricas de los equipos para todo el CRTV, Todos los parámetros definidos en el presente numeral se encuentran en el anexo adjunto, en la que es realizada por el municipio del Cantón Cañar, considerando que la institución cuenta en la actualidad con un crédito aprobado por el BDE, en la que se hace constar los términos técnicos y factores económicos que necesita el centro de revisión técnica vehicular.

La tabla 10 nos muestra las alturas y anchos mínimos que debe respetar los CRTV para que puedan entrar en funcionamiento.

Tabla 14.

Alturas y anchos mínimos para CRTV.

\begin{tabular}{|l|l|l|}
\hline Clasificación & Altura mínima & Ancho mínimo \\
\hline Para el CDA sea en la clase C o de tipo D & $4,5 \mathrm{~m}$ & $4,5 \mathrm{~m}$ \\
\hline Para el CDA sea en la clase B & $4,5 \mathrm{~m}$ & $4,0 \mathrm{~m}$ \\
\hline Para el CDA sea en la clase A & $4,5 \mathrm{~m}$ & $3,0 \mathrm{~m}$ \\
\hline
\end{tabular}

\section{DIMENSIONES PROPUESTAS PARA LAS LÍNEAS DE RTV DE LA EPPC}

De acuerdo con lo analizado en los puntos anteriores y respetando las normas locales e internacionales se plantea las siguientes dimensiones para el CRTV:

Se considera una longitud de línea de $25 \mathrm{~m}$ con un ancho mínimo de $5 \mathrm{~m}$ para livianos Se considera una longitud de línea de $40 \mathrm{~m}$ con un ancho mínimo de $6 \mathrm{~m}$ para pesados Se considera una longitud de línea de $12 \mathrm{~m}$ con un ancho mínimo de $3 \mathrm{~m}$ para motocicletas Con un área estimada total de $780 \mathrm{~m}^{2}$ 


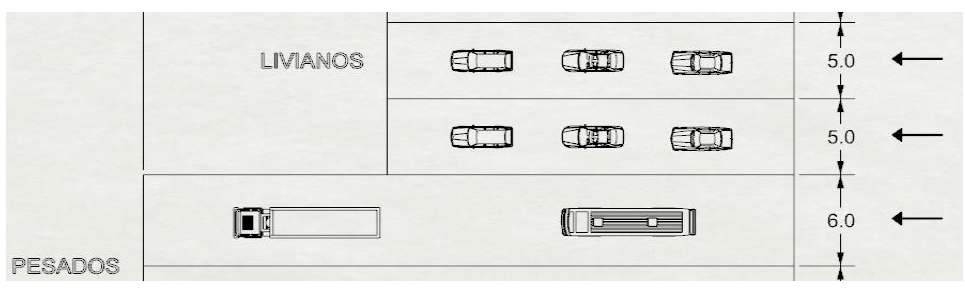

Figura 3. Dimensiones propuestas CRTV.

\section{ESTRUCTURA OPERATIVA DE LAS LINEAS DE REVISIÓN TÉCNICA VEHICULAR}

Toda la gestión administrativa y técnica que se ve involucrada dentro del centro de revisión técnica contempla inicialmente una comprobación documental legal del automotor en turno que consiste principalmente de: cancelación de rubros de impuestos, contratos de compra - venta necesariamente notariados entre otros más; posterior a esta etapa viene las inspección técnica visual y mecánica integral del automotor acorde a la norma legal vigente, el resultado de éste procedimiento se plasma sobre un informe final que contendrá todos los pormenores acerca de la condición específica del vehículo con esto hacer la emisión respectiva de un certificado aprobando o no al vehículo bajo análisis su libre circulación, promoviendo seguridad para peatones, pasajeros, y que los humos provenientes de su combustión no causa daños al ambiente.

Se puede demostrar en el gráfico que se muestra a continuación, mediante un diagrama de flujo el procedimiento y etapas que son realizadas en una revisión de tipo técnica en el vehículo. 


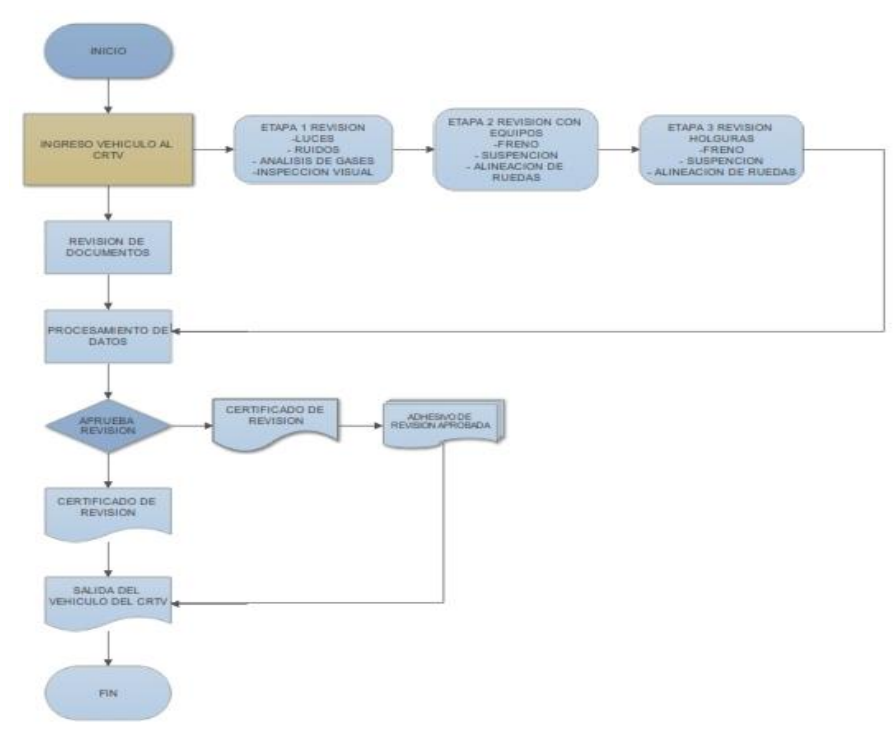

Figura 4. Diagrama del Proceso Revisión Vehicular

\section{CONCLUSIONES}

De acuerdo a las encuestas realizadas a la ciudadanía del cantón cañar pudimos observar que muchas de las personas no conoce el beneficio social, ambiental que brindara este centro de revisión técnica vehicular para lo cual se deberá socializar los procedimientos a los cuales se someterán sus vehículos y con ello cual será el beneficio para sus conductores y a las personas que hagan uso del transporte público del cantón. Por qué el beneficio no es solo para los conductores sino para toda la sociedad en general debido al control que va ejercer este centro RTV sobre la calidad de aire de los vehículos y así proteger el medio ambiente para futuras generaciones.

De acuerdo con las encuestas realizadas sobre el proyecto de CRTV ubicado en la ciudad de Cañar. Se determinó que es necesario tres líneas de revisión para RTV, dos líneas para vehículos livianos y una línea mixta. 


\section{REFERENCIAS CONSULTADAS}

ANT. (2015). Ley Organica Reformatoria a la Ley organica de Transporte Terrestre, Transito y Seguridad Vial. Obtenido de Agencia Nacional de Transito. https://n9.cl/n3h4

Asamblea Nacional. (2017). Resolución No. 063 Reforma a la Resolución No. 070-DIR2015-ANT que contiene el "Reglamento Relativo a los Procesos de la Revisión de Vehículos a Motor". https://n9.cl/jaqb0

Chiavenato, I. (1999). Administracion de Recursos Humanos. Mc Graw Hill.

Daft, R. (2011). Teoría y diseño. México, D.F: CENGAGE Learning.

Erazo, J. C., \& Narváez, C. I. (2020). La gestión del capital intelectual y su impacto en la efectividad organizacional de la industria de cuero y calzado en la Provincia de Tungurahua - Ecuador [The management of intellectual capital and its impact on the organizational effectiveness of the leather and footwear industry in the Province of Tungurahua - Ecuador]. Revista Espacios, 41(21), 254-271.

Fernández-Solís, C. (2018). Propuesta de diseño de una estructura organizacional para la coordinación zonal 5 de educación. Recuperado de https://n9.cl/4ofv0

Gareth R., J. (2013). Teoría organizacional. México: Pearson Education Mexico.

Melinkoff, R. (1969). La Estructura de la Organizacion. Caracas: Universidad Central de Venezuela.

Merton, R. K. (1968). Social Theory and Social Structure. Nueva York: The Free Press.

Minsal-Pérez, D, \& Pérez-Rodríguez, Y. (2007). Organización funcional, matricial: En busca de una estructura adecuada para la organización. ACIMED, 16(4)

Mintzberg, H. (2016). La configuración organizacional: el modelo de Mintzberg. Obtenido de https://n9.cl/irr1j

N3422-2004. Resolución Directorial (2004). Tabla de infraestructura y equipamiento mínimo para plantas y revisiones técnicas vehiculares. Lima. https://n9.cl/adewj 


\section{CIENCIAMATRIA}

Revista Interdisciplinaria de Humanidades, Educación, Ciencia y Tecnología

Año VII. Vol. VII. N¹2. Enero - Junio. 2021

Hecho el depósito de ley: pp201602FA4721

ISSN-L: 2542-3029; ISSN: 2610-802X

Universidad Nacional Experimental Francisco de Miranda (UNEFM). Santa Ana de Coro. Venezuela

Ángel Sebastián Martínez-Narváez; Juan Carlos Erazo-Álvarez; Cecilia Ivonne Narváez-Zurita Cristián Andrés Erazo-Álvarez

Naranjo García, D. D. (2014). Análisis y propuesta estratégica de un nuevo modelo de gestión del servicio de matriculación vehicular orientado al mejoramiento de la atención a los usuarios dentro del Distrito Metropolitano de Quito. UIDE, 15-16.

NTE INEN 2 207:2002. Gestión ambiental. Aire. Vehículos automotores. Límites permitidos de emisiones producidas por fuentes móviles terrestres de diésel. https://n9.cl/8z93

Psicologia Online. (2018). Estructura Simple. Obtenido de https://n9.cl/j7vuw

Publicaciones Vértice S.L. (2008). Estructuras organizativas. Recuperado de https://n9.cl/f8e4

Robbins S. (2005). Administration. Pearson education Inc.

(C2021 por los autores. Este artículo es de acceso abierto y distribuido según los términos y condiciones de la licencia Creative Commons Atribución-NoComercial-Compartirlgual 4.0 Internacional (CC BY-NC-SA 4.0) (https://creativecommons.org/licenses/by-nc-sa/4.0/). 